\title{
Evaluating the Acceptability and Feasibility of Providing Egg or Cereal Breakfast during a Family-Based Treatment for Children with Overweight/Obesity: The Families and Breakfast Pilot Trial
}

\author{
Kerri N. Boutelle, PhD, ${ }^{1-3}$ Michael A. Manzano, BS,'4 David R. Strong, PhD,2 \\ and Kyung E. Rhee, MD, MSc, MA'
}

\section{Abstract}

Background: Family-based behavioral treatment (FBT) is the most successful weight-loss treatment for children with overweight and obesity, however, long-term success is only achieved by a third of children over time. The use of foods that induce satiety, such as eggs, could improve adherence to calorically restricted diets in children and improve outcomes. This study explored the consumption of eggs (FBT+egg) or cereal (FBT+cereal) for breakfast as part of an FBT program, when breakfast foods were provided to families.

Methods: Fifty 8-12-year-old children with overweight and obesity and their parents were randomized to a 4-month FBT+egg or FBT+cereal treatment program. Families were provided the ingredients for their assigned breakfast at each treatment session, and instructed to consume the breakfast a minimum of 5 days per week. Families attended assessments at baseline, post-treatment, and 4months post-treatment.

Results: Results showed that both treatments were well liked, FBT attendance was similar, and there was high compliance with consumption of the specified breakfast. Children experienced moderate weight loss at post-treatment [-0.11 standardized BMI (BMIz)] through 4-month follow-up (-0.09 BMIz), with no statistically significant differences (mean difference $-0.05 \mathrm{BMI}, 95 \%$ confidence interval -0.19 to 0.09 ) observed between egg and cereal conditions across any anthropometric or appetitive measures.

Conclusions: The use of eggs for breakfast in children enrolled in FBT was well tolerated, and future studies should include larger samples and longer follow-up periods to assess the potential differential effects of prescribed breakfasts on children's weight and eating behaviors.

Keywords: breakfast; cereal; child; egg; overweight; obesity; pediatric; weight loss

\section{Background}

$\mathbf{R}$ ecent data suggest that $35 \%$ of children in the United States have overweight or obesity, affecting 4-5 million children in the United States. ${ }^{1}$ Children who have overweight or obesity are at an increased risk for many negative health sequelae in childhood and adulthood, including orthopedic and endocrine conditions, cardiovascular disease, cancer, and all-cause mortality. ${ }^{2-8}$ Children who are obese compared to healthy weight, have significantly higher health care costs, including a higher frequency of outpatient and emergency room visits. ${ }^{9}$
The current model for the treatment of childhood obesity is family-based behavioral treatment (FBT), which combines nutrition and exercise education with behavior therapy techniques. ${ }^{10-12}$ FBT recommends the Traffic-light diet, which categorizes foods into the three colors of the traffic light on the basis of energy density. With the exception of discouraging fats and sugars due to their energy density, FBT does not make specific dietary recommendations based on nutrient composition, and families are directed to focus on reducing total energy intake. With regards to exercise, children are excouraged to participate in 90 minutes of physical activity at least 5 days/week.

Departments of 'Pediatrics, ${ }^{2}$ Family Medicine and Public Health, and ${ }^{3}$ Psychiatry, University of California, San Diego, La Jolla, CA.

${ }^{4}$ San Diego State University/University of California, San Diego Joint Doctoral Program in Clinical Psychology, San Diego, CA. 
Although FBT is considered the most effective model, ultimately two-thirds of children fail to achieve sustained weight loss over time. ${ }^{13}$ It is possible that the restricted calorie diet prescribed in FBT is not sustainable by the children over time in its current form.

Despite the assertion that "all calories are equal," a substantial literature suggests that completion of weight loss interventions and weight loss is higher among participants who were assigned to diets with higher ratio of protein to carbohydrates, compared to diets that have higher ratios of carbohydrates to protein. ${ }^{14}$ Over the past few decades, there have been studies that demonstrate the differential effects of macronutrients on body mass, independent of their energy values. ${ }^{15}$ Studies have shown that differences in macronutrient composition result in varying level of thermogenesis and fat storage. ${ }^{16}$ Advances in our understanding of macronutrient composition on weight loss have not been applied to children with overweight or despite the high prevalence rates and limited success of treatments using caloric restriction as the primary premise for weight loss.

One method to potentially improve outcomes in FBT is to use a more sophisticated dietary approach that is based on the satiating properties of the diet. ${ }^{17}$ Studies have shown that individuals endorse higher levels of satiety and lower levels of hunger after consuming foods high in protein in comparison to foods comprised mainly of carbohydrates, fat, and/or sugar. ${ }^{16}$ Since children with obesity may be at heightened risk for overconsumption under the conditions of calorie restriction, prescribing daily consumption of foods that have appetite-regulating properties could reduce the risk of overeating and lead to greater success in achieving sustained weight loss.

Eggs have a significantly greater satiety index than other breakfast foods including ready-to-eat cereals and white bread ${ }^{18}$ and could reduce overall caloric intake. ${ }^{19}$ Eggs are high in leucine, which has been shown to promote greater satiety by differentially affecting hormones that modulate satiety and food intake, including insulin, ghrelin, and glucagon. ${ }^{20}$ Compared to other branch-chained amino acids, leucine passes the blood-brain barrier more quickly and engages neurocircuitry, which is implicated in the regulation of food intake, specifically the hypothalamusbrain stem circuit. ${ }^{20,21}$ In adult studies, the use of an egg breakfast during a calorie-restricted weight loss diet showed greater reductions in weight loss compared to a bagel breakfast matched for energy density and total energy. ${ }^{22}$ Studies evaluating the short-term effects of an egg breakfast on satiety in adults showed that individuals experience greater satiety, less hunger, and a lower desire to eat after consuming an egg breakfast compared to breakfasts weighted toward higher carbohydrate compositions (cereals, breads). ${ }^{23}$ Accordingly, in child studies comparing breakfasts of varying macronutrient compositions, children consumed less calories when given an egg breakfast and reported feeling less hungry at lunchtime in comparison to a cereal breakfast. ${ }^{24}$ These findings suggest that an egg breakfast may promote greater satiety and influence total energy intake in children, which could be beneficial to children on a restricted energy diet for weight loss.

Thus, the primary aims of this study were to evaluate the feasibility, compliance, and tolerance of recommending eggs or cereal for breakfast as part of FBT pilot treatment. Secondary aims include comparing the changes in process measures, including child and parent anthropometrics; child eating and appetitive traits; and blood glucose, triglycerides, and cholesterol at post-treatment and 4-months post-treatment.

\section{Methods}

\section{Study Design}

The Families and Breakfast (FAB) study was a pilot randomized controlled trial that evaluated two 4-month treatments for childhood obesity: FBT+egg breakfast and FBT+cereal breakfast. The only difference between the two arms was which breakfast was assigned. Both treatments included nutrition and physical activity recommendations, parenting skills, and behavior modification strategies. Additionally, families were provided the ingredients for their assigned breakfast and were instructed to consume the breakfast a minimum of 5 days per week. Both groups were led on the same night of the week with the same group leaders who attended weekly supervision with the first author. Measures were collected at baseline, post-treatment, and 4-month follow-up. Once sufficient numbers of participants were accrued, individuals were randomized and allocated to either egg or cereal conditions utitilzing within-cohort randomization to ensure group sizes were equivalent each cohort. The primary aims evaluated group attendance, compliance with breakfast consumption, and toleration of the prescribed breakfast. Secondary aims included comparing the changes in process measures, including child and parent anthropometrics, child eating in the absence of hunger, child power of food, child food cravings as well as blood glucose, triglycerides, and cholesterol at post-treatment and 4-months posttreatment. The Institutional Review Boards of University of California, San Diego, and Rady Children's Hospital, San Diego, California approved the study. Written consent and assent was obtained from parents and children, respectively.

\section{Eligibility and Recruitment}

Eligibility included a child between 8.0 and 12.9 years of age with a BMI between the 85th and 99.9th percentiles, a parent in the household with a BMI of at least 25 who could read English at a minimum of a fifth-grade level, child endorsement of liking of both eggs and cereal, and availability to participate in the study on designated evenings. Exclusionary criteria included a major child or parent psychiatric disorder, child diagnosis of a serious current physical disease, child with physical limitations, 
child dislike of eggs or cereal, or a family with food restrictions. The blood draw was optional for the families. Children with overweight or obesity and their parent were recruited through primary care physicians, schools, listserves, local advertisements, and advertisements. One hundred fifty-one families contacted the Center for Healthy Eating and Activity Research (CHEAR) for the FAB study, 64 attended a baseline assessment and 50 child-parent dyads were randomized. Of note, two families reported that their child would not eat eggs or cereal, and were not randomized. Child-parent dyads were randomly assigned to either the FBT+egg or FBT+cereal arm stratified by sex of the child by the data manager.

\section{Intervention}

The treatment programs included 16 visits over 4 months, and the content was based on published trials of FBT. ${ }^{11,12}$ Parents and children in both arms attended simultaneous 1-hour parent or child groups. Families were instructed to eat their prescribed breakfast (egg or cereal) 5 days of the week during the 4-month treatment program, but were provided with enough of the prescribed breakfast to eat it all 7 days if desired. In an attempt to minimize difficulties with compliance, children rated their liking of eggs and cereal (Methods section) at the baseline assessment and families were provided with enough of their assigned breakfast each week for both the participating parent and child to consume. Families were provided recipes for both egg and cereal arms that matched the breakfasts on total calories; however, the egg breakfast provided a higher proportion of calories than from protein and fat. Families in the egg condition were given 30 eggs, 0.5 gallon of milk ( $2 \%$ or soy), 7 applesauce or peach fruit cups for the child and 7 applesauce or peach fruit cups for the parent each week, and were told to consume 2 eggs, $1 / 2$ cup milk, and 1 fruit cup as a breakfast serving. The macronutrient profile of the prescribed egg breakfast was 16 gram protein, 27 gram carbohydrate, and 13 gram fat. Families in the cereal condition were given 1 box of preferred cereal (corn flakes, Cheerios, or Kix), 1.5 gallons of milk ( $2 \%$ or soy), 7 applesauce or peach fruit cups for the child and 7 applesauce or peach fruit cups for the parent each week, and were told to consume 1 cup of cereal with $1 \frac{1}{4}$ cup of milk and 1 fruit cup as a breakfast serving. The macronutrient profile of the prescribed cereal breakfast was 12 gram protein, 59 gram carbohydrate, and 6 gram fat.

\section{Measures}

Assessments with child-parent dyads were conducted at baseline, post-treatment and 4-month follow-up. Data collection was conducted by trained staff and supervised by $\mathrm{PhD}$-level psychologists. Participants received incentives for time, travel, and effort at assessments.

Attendance, compliance, and tolerance. Session attendance and program attrition were recorded by study staff. At each treatment session parents were asked how many days the prescribed breakfast was consumed during the prior week. Acceptability was assessed immediately following completion of the intervention using questions designed specifically for this study. Parents responded to questions regarding how much they liked the program ("How much did you like the group overall?") and the convenience of their assigned group ("How convenient was the group you were assigned?")

Taste test. Children were asked to taste cereal, milk, and eggs and rate each on a 1-5 likert type scale, with " 1 " indicating disgusting and " 5 " indicating "delicious."

Anthropometry. Height and weight were measured in duplicate. The mean of the two values was used to calculate BMI (calculated as weight in kilograms divided by height in meters squared). For children, age-adjusted BMI percentile (BMI\%) and standardized BMI (BMIz) were calculated..$^{25}$

Child Power of Food Scale. The Child Power of Food Scale (C-PFS) is a 15-item measure to assess children's perceptions of the power of food at different levels of proximity. ${ }^{26}$ Only the food present (FP) subscale was included in this study. Scores from the C-PFS-FP subscale demonstrated adequate reliability $(\alpha=0.77 ; \omega=0.86)$.

Eating in the Absence of Hunger Questionnaire. The Eating in the Absence of Hunger Questionnaire (EAHQ) is a 14-item measure to assess children's perceptions of the frequency with which they ate in response to a variety of moods and environments while sated. ${ }^{27}$ Scores from the EAHQ demonstrated good reliability $(\alpha=0.91$; $\omega=0.95)$.

Food Craving Questionnaire-Trait. The Food Craving Questionnaire-Trait (FCQ-T) is a 39-item measures to assess children's perceptions of the factors leading up to and following food cravings. ${ }^{28}$ A 37 -item abridged version was administered to children, as two items ("When I'm stressed out, I crave food" and "I crave foods when I'm upset") were deemed developmentally inappropriate for the current sample by the study team. Scores from the FCQ-T demonstrated good reliability $(\alpha=0.95 ; \omega=0.97)$.

Glucose, triglycerides, and cholesterol. Children provided a fasting blood sample for glucose, triglycerides, and cholesterol at the UCSD Altman Clinical and Translational Research Institute.

Demographics. Race, ethnicity, gender, and family income for both the parent and child were reported by the parent during the families' baseline assessment.

\section{Statistical Analysis}

The primary analyses were performed for the intentionto treat population, defined as the child-parent dyads who were allocated to either FBT+egg or FBT+cereal arm. 
Demographic information, treatment attendance, and acceptability measures were compared using independent sample $t$-tests or chi-squared tests where appropriate. Linear mixed effects regression models were used for all other outcome analyses, with models controlling for the planned covariates of age and gender. Cook's distance calculations were used to identify overly influential data, with cases greater than four times the mean Cook's distance of the model being excluded from outcome analyses. All analyses were run using $\mathrm{R}$ version 3.4.3.

\section{Results}

\section{Participants}

We screened by telephone 151 families who expressed interest, conducted assessments with 64 child-parent dyads, and enrolled 50 parent/child dyads (Fig. 1). One family did not attend treatment after randomization. Similar baseline characteristics were observed in both FBT-egg and FBT-cereal (Table 1). Of the parent/child dyads enrolled, data from $72 \%(n=36)$ were available at post-treatment and data from $60 \%(n=30)$ were available at 4-month post-treatment. Consent for the blood draw was optional, and 33 families provided samples at baseline and 23 at post-treatment.

\section{Primary Outcomes}

Attendance, compliance, and tolerance. Treatment attendance was similar between the egg and cereal conditions $[$ egg $=9.64(5.15)$ and cereal $=8.68(5.74)]$. Retention at both post-treatment $(\mathrm{egg}=80 \%$; cereal $=64 \%)$ and 4-month follow-up (egg $=68 \%$; cereal $=52 \%)$ assessments was not significantly different between the groups but trended higher

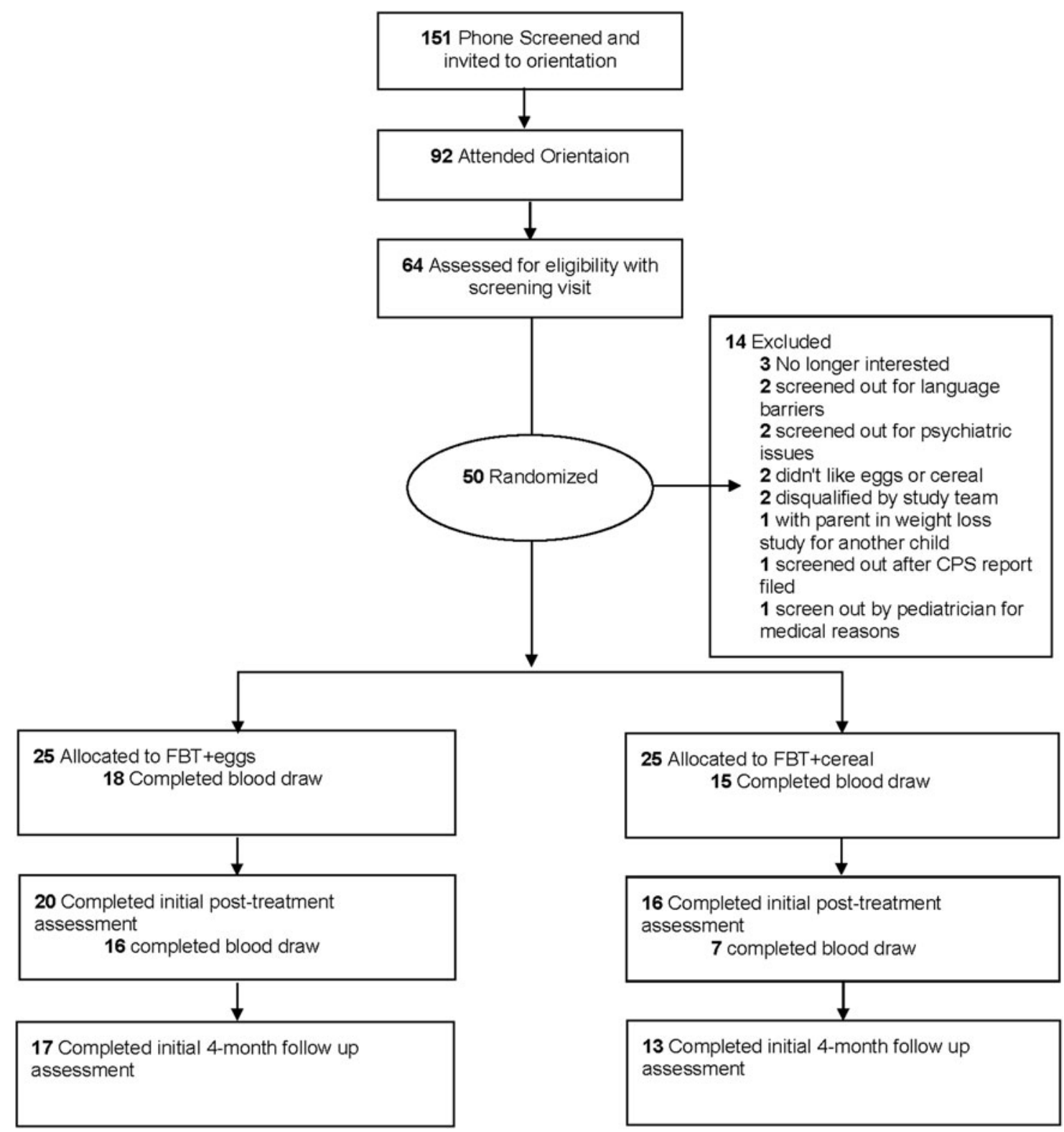

Figure 1. Consort diagram describing study recruitment, treatment, and assessment flow. 
Table I. Sample Characteristics

\section{\begin{tabular}{l|c|c} 
Total sample, $\mathbf{N}=\mathbf{5 0}$ & FBT-egg, $\mathbf{N}=\mathbf{2 5}$ & FBT-cereal, $\mathbf{N}=\mathbf{2 5}$
\end{tabular}}

$t$-Test/chi-squared test

Child

\begin{tabular}{|c|c|c|c|c|}
\hline \multicolumn{5}{|l|}{ Age, mean (SD) } \\
\hline Years & $10.86(1.45)$ & $11.08(1.37)$ & $10.64(1.51)$ & $1.08(p=0.29)$ \\
\hline \multicolumn{5}{|l|}{ Gender } \\
\hline Female & $56 \%$ & $54 \%$ & $60 \%$ & $0.08(p=0.77)$ \\
\hline \multicolumn{5}{|c|}{ Body size, mean (SD) } \\
\hline $\mathrm{BMI}$ & $27.53(4.58)$ & $28.70(4.33)$ & $26.4 I(4.6 I)$ & $1.78(p=0.08)$ \\
\hline BMI percentile & $96.90(3.36)$ & $97.59(2.84)$ & $96.23(3.73)$ & $0.46(p=0.64)$ \\
\hline BMlz & $2.03(0.42)$ & $2.13(0.38)$ & $1.94(0.43)$ & $0.99(p=0.33)$ \\
\hline \multicolumn{5}{|l|}{ Race/ethnicity } \\
\hline Hispanic & $64 \%$ & $72 \%$ & $56 \%$ & \multirow[t]{5}{*}{$3.40(p=0.18)$} \\
\hline Non-Hispanic & \multirow[t]{2}{*}{$10 \%$} & \multirow[t]{2}{*}{$12 \%$} & \multirow[t]{2}{*}{$8 \%$} & \\
\hline Other & & & & \\
\hline Non-Hispanic & \multirow[t]{2}{*}{$26 \%$} & \multirow[t]{2}{*}{$16 \%$} & \multirow[t]{2}{*}{$36 \%$} & \\
\hline White & & & & \\
\hline \multicolumn{5}{|l|}{ Parent } \\
\hline \multicolumn{5}{|l|}{ Age, mean (SD) } \\
\hline Years & $41.02(7.53)$ & $39.92(7.88)$ & $42.17(7.14)$ & $-1.05(p=0.30)$ \\
\hline \multicolumn{5}{|l|}{ Gender } \\
\hline Female & $86 \%$ & $92 \%$ & $80 \%$ & $0.66(p=0.42)$ \\
\hline \multicolumn{5}{|c|}{ Body size, mean (SD) } \\
\hline $\mathrm{BMI}$ & $32.33(10.03)$ & $31.84(10.49)$ & $32.83(9.73)$ & \\
\hline \multicolumn{5}{|l|}{ Marital status } \\
\hline Married & $76 \%$ & $72 \%$ & $80 \%$ & \multirow[t]{2}{*}{$5.44(p=0.36)$} \\
\hline Divorced/single & $28 \%$ & $16 \%$ & $12 \%$ & \\
\hline \multicolumn{5}{|l|}{ Race/ethnicity } \\
\hline Hispanic & $54 \%(27)$ & $64 \%(16)$ & $44 \%(11)$ & \multirow[t]{5}{*}{$3.82(p=0.15)$} \\
\hline Non-Hispanic & \multirow[t]{2}{*}{$14 \%(7)$} & \multirow[t]{2}{*}{$16 \%(4)$} & \multirow[t]{2}{*}{$12 \%(3)$} & \\
\hline Other & & & & \\
\hline Non-Hispanic & \multirow[t]{2}{*}{$32 \%(16)$} & \multirow[t]{2}{*}{$20 \%(5)$} & \multirow[t]{2}{*}{$44 \%(I I)$} & \\
\hline White & & & & \\
\hline \multicolumn{5}{|l|}{ Household income } \\
\hline$>100,000$ & $34 \%(17)$ & $28 \%(7)$ & $40 \%(10)$ & $8.86(p=0.63)$ \\
\hline
\end{tabular}

FBT, family-based behavioral treatment.

in the egg condition over the cereal condition. At baseline, children reported liking the cereal significantly more then the eggs (cereal liking $=4.42$, eggs liking $=4.04 ; t=2.16$; $p=0.036 ;-0.35$, confidence interval $[95 \% \mathrm{CI}] 0.03-0.73)$. At post-treatment, the weekly rates of child consumption of the prescribed breakfast were similar between the egg and cereal condition $[\mathrm{egg}=5.88$ days $(1.59)$; cereal $=5.64$ days $(1.21) ; t(24)=0.44, p=0.66]$ and were higher than the 5 days prescribed. There were no differences in parent reports of likability $[t(21)=0.81, p=0.43]$ or convenience 
$[t(21)=0.36, p=0.73]$ between groups, with the majority of parents in both groups $(\mathrm{egg}=81 \%$; cereal $=91 \%)$ reporting they "very much liked" or "somewhat liked" their group. There were no significant differences between those who completed the treatment and those who $\operatorname{did} \operatorname{not}(p$ 's $>0.10)$.

\section{Secondary Outcomes}

Child and parent weight. Across groups, children experienced moderate weight loss at post-treatment $(-0.11 \mathrm{BMIz}$; $d=-0.37,95 \% \mathrm{CI}-0.81$ to 0.07 ) and 4-month follow-up $(-0.09 \mathrm{BMIz} ;=-0.42,95 \% \mathrm{CI}-0.89$ to 0.05 ; Fig. 1 ; top). There were no significant differences in weight loss assessed with child BMIz $[F(1,24)=0.48, p=0.50]$ or parent BMI $[F(1,24)=0.60, p=0.44]$ between the egg and cereal conditions at post-treatment and 4-month follow-up. Across groups, parents experienced negligible weight loss at post-treatment $(d=-0.07,95 \% \mathrm{CI}-0.52$ to 0.38$)$ and 4-month post-treatment ( $d=-0.04,95 \%$ CI -0.45 to 0.52 ; Fig. 2 ; bottom).

Child Power of Food-Food Present Subscale. There were no significant differences in child self-reported PFSFP between the egg and cereal conditions at post-treatment and 4-month post-treatment $[F(1,20)=0.18, p=0.68]$. Across groups, children experienced small changes in PFS-FP at post-treatment $(d=-0.15,95 \% \mathrm{CI}-0.60$ to $0.31)$ and 4-month post-treatment $(d=-0.30,95 \% \mathrm{CI}$ -0.80 to 0.21 ; Fig. 3 ).
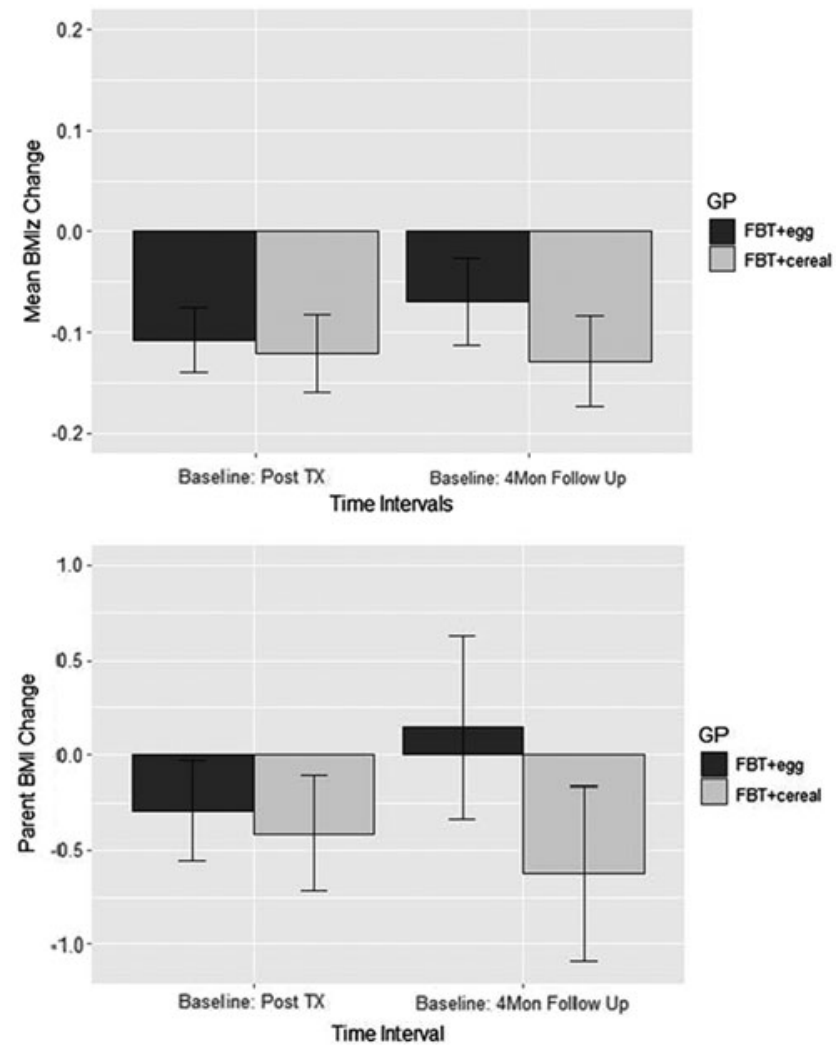

Figure 2. Differences in child weight (BMIz; top) and parent weight (BMI; bottom) at post-treatment and 4-month follow-up. BMIz, standardized BMI; FBT, family-based behavioral treatment; GP.

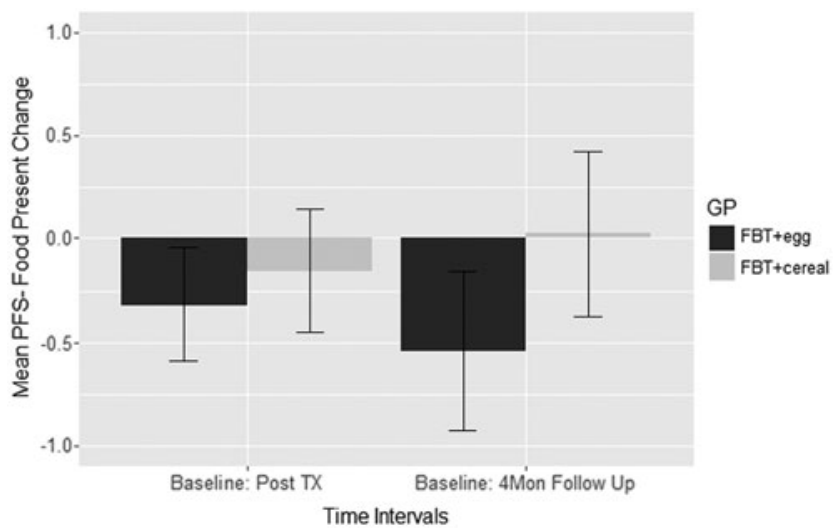

Figure 3. Changes in child Power of Food-Food Present Subscale from baseline to post-treatment and 4-month follow-up. PFS, Power of Food Scale.

Eating in the Absence of Hunger Questionnaire. There were no significant differences in child self-reported EAHQtotal score between the egg and cereal conditions at posttreatment and 4-month post-treatment $[F(1,18)=0.70$, $p=0.41]$. Across groups, children experienced negligible changes in eating in the absence of hunger at post-treatment $(d=-0.12,95 \% \mathrm{CI}-0.64$ to 0.40$)$ and moderate changes at 4-month post-treatment $(d=-0.49,95 \% \mathrm{CI}-1.12$ to 0.14 ; Fig. 4).

Food Craving Questionnaire-Trait. There were no significant differences in child self-reported FCQ between the egg and cereal conditions at post-treatment and 4month post-treatment $[F(1,20)=1.75, p=020]$. Across groups, children experienced negligible changes in subjective cravings at post-treatment $(d=-0.07,95 \% \mathrm{CI}-0.57$ to 0.43$)$ and at 4 -month post-treatment $(d=-0.16,95 \% \mathrm{CI}$ -0.75 to 0.44 ; Fig. 5 ).

Glucose, tricylcerides, and cholesterol. There were no significant differences in fasting glucose $[F(1,18)=0.19$, $p=0.67]$, triglycerides $[F(1,19)=1.22, p=0.28]$, or total

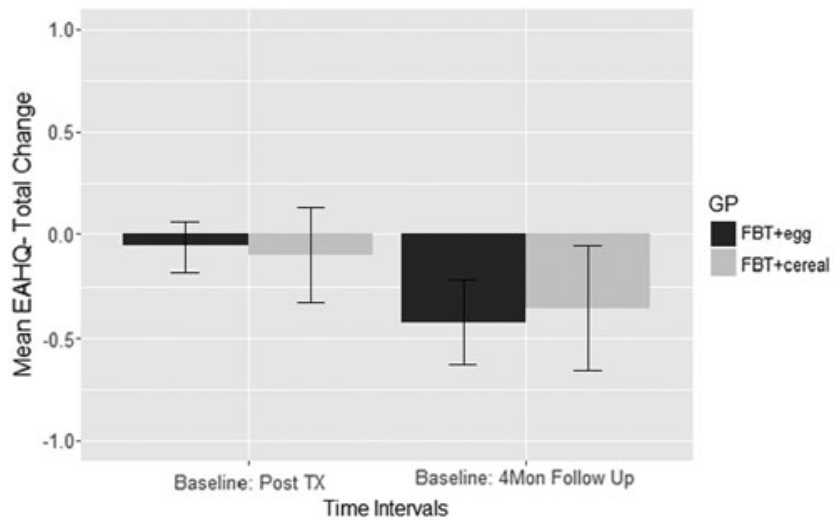

Figure 4. Changes in child Eating in the Absence of Hunger Scale from baseline to post-treatment and 4-month follow-up. EAHQ, Eating in the Absence of Hunger Questionnaire. 


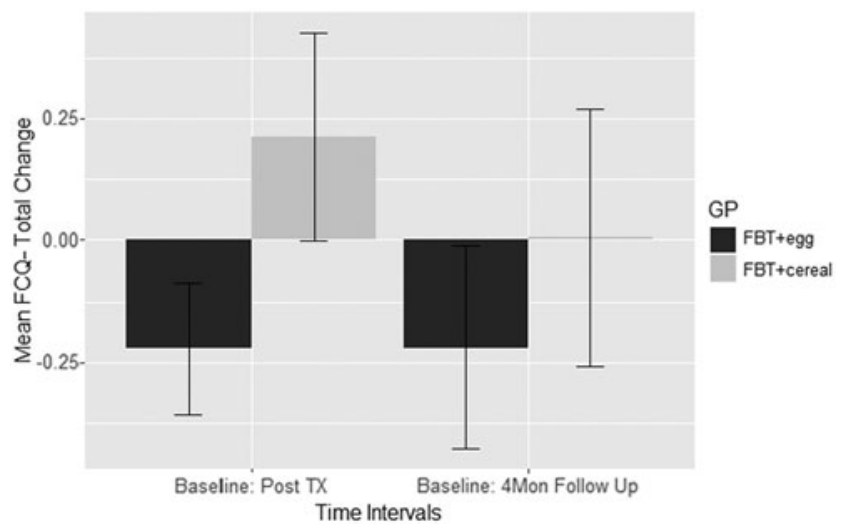

Figure 5. Changes in child FCQ from baseline to post-treatment and 4-month follow-up. FCQ, Food Craving Questionnaire.

cholesterol $[F(1,18)=0.06, p=0.81]$ between groups at post-treatment. Across groups, children experienced moderate changes in fasting glucose $(d=-0.42,95 \% \mathrm{CI}-0.97$ to $0.14)$, negligible changes in cholesterol $(d=0.09,95 \% \mathrm{CI}$ -0.46 to 0.64 ), and negligible changes in triglycerides $(d=0.04,95 \%$ CI -0.51 to 0.58$)$.

\section{Discussion}

To our knowledge, this is the first evaluation of the use of a high protein breakfast prescription for children as part of a 4-month FBT intervention. The attendance and acceptability data were similar for the FBT+egg group and the FBT+cereal group, suggesting that utilizing eggs for breakfast did not impact the provision of the intervention. Likeability of the intervention, attendance, adherence, and retention were also similar between the two arms. However, it should be noted that children rated their liking of the cereal significantly more than the eggs at baseline. Anecdotally, it was noted by recruitment staff that some families were screened out because they said their child would not consume eggs 5 days a week.

Overall, both arms, FBT+egg and FBT+cereal, showed significant but moderate decreases in child weight (BMIz score) immediately after treatment and these changes were maintained at the 4-month follow-up. There were no significant differences between the FBT+egg and FBT+cereal groups for child BMIz score or parent BMI at either posttreatment or at 4-month follow-up. There were no significant changes in glucose, cholesterol, or triglycerides between the two groups, suggesting a potential lack of negative effects of consuming eggs for breakfast 5 days a week in 8-12-year-old children.

We predicted that having eggs for breakfast would increase a child's satiety, which could impact how they interact with food and reduce overeating. Similar to other outcomes in this study, there were no significant differences in the child's responsiveness to food cues, child's eating in the absence of hunger, or child's cravings. Although nonsignificant, these data indicate that children in the egg arm may have had larger decreases in food responsiveness, eating in the absence of hunger, and cravings. Larger studies are needed to explore how consuming eggs for breakfast impact these important variables in children over time.

Although the lack of differences between the two groups suggests that there are no negative effects of prescribing eggs for breakfast in 8-12-year-old children, it is also important to note that there were no significant benefits identified either. There are a few factors that may have impacted the failure to reject the null hypothesis in this trial. This is a small pilot study that was underpowered to detect differences between two active treatment groups. The treatment in this study was provided over 4-months, and it is possible that differences may have emerged if the treatment was 6-months similar to other FBT trials. ${ }^{23}$ Although we used an intent-to-treat approach for analyses, the drop-out rate may have impacted the variability in measures. It is also possible that more sensitive measures would have identified changes in satiety throughout the treatment programs.

Strengths of the study include the acceptability data, randomized design, racial/ethnic diversity of the families, and use of a validated treatment protocol. As in all studies, there are limitations that need to be considered. This study included a 4-month follow-up and a modest sample size, and larger studies are needed to provide fully powered efficacy data. Study participants were treatment-seeking volunteers with 8-12-year-old children with overweight or obesity, limiting the generalizability. Additionally, this study did not include a placebo control intervention. In sum, this study demonstrated the feasibility and acceptability of prescribing a high protein egg breakfast for 8 12-year-old children in the context of FBT. Future studies with larger samples and more sensitive measures are needed to evaluate any differences between the impact of high protein and high carbohydrate breakfasts on outcomes in FBT.

\section{Acknowledgment}

Funding provided by the American Egg Board/Egg Nutrition Center.

The authors support data sharing. All data summarized in this publication may be available in a deidentified format to other investigators for research purposes with the approval of the principal investigators and the Institutional Review Board immediately after the publication of this article.

\section{Author Disclosure Statement}

No competing financial interests exist.

\section{References}

1. Skinner AC, Ravanbakht SN, Skehon, JA, et al. Prevalence of obesity and severe obesity in US children, 1999-2016. Pediatr 2018;141:e20173459. 
2. Daniels S. The consequences of childhood overweight and obesity. Future Child 2006;16:47-67.

3. Dietz W. Health consequences of obesity in youth: Childhood predictors of adult disease. Pediatrics 1998;101 (3 Pt 2):518-525.

4. Dixon J. The effect of obesity on health outcomes. Mol Cell Endocrinol 2010;316:104-108.

5. Gunnell D, Frankel S, Nanchahal K, et al. Childhood obesity and adult cardiovascular mortality: A 57-y follow-up study based on the Boyd Orr cohort. Am J Clin Nutr 1998;67:1111-1118.

6. Reilly J, Kelly J. Long-term impact of overweight and obesity in childhood and adolescence on morbidity and premature mortality in adulthood: Systematic review. Int J Obes (Lond) 2011;35:891898.

7. Sorof J, Daniels S. Obesity hypertension in children: A problem of epidemic proportions. Hypertension 2002;40:441-447.

8. Key T, Schatzkin A, Willett W, et al. Diet, nutrition and the prevention of cancer. Pub Health Nutr 2004;7(1A):187-200.

9. Janicke D, Harman J, Jamoom E, et al. The relationship among child weight status, psychosocial functioning, and pediatric health care expenditures in a medicaid population. $J$ Pediatr Psychol 2010;35:883-891.

10. Epstein L. Development of evidence-based treatments for pediatric obesity. In: Kazdin A, Weisz J (eds). Evidence-Based Psychotherapies for Children and Adolescents. Guilford Publications: New York, 2003, pp. 374-388.

11. Boutelle K, Rhee K, Liang JB, A, et al. Effect of attendance of the child on body weight, energy intake, and physical activity in childhood obesity treatment: A randomized clinical trial. JAMA Pediatr 2017;171:622-628.

12. Wilfley D, Tibbs T, Van Buren D, et al. Lifestyle interventions in the treatment of childhood overweight: A meta-analytic review of randomized controlled trials. Health Psychol 2007;26: 521-532.

13. Epstein L, Valoski A, Wing RR, et al. Ten-year follow-up of behavioral, family-based treatment for obese children. JAMA 1990; 264:2519-2523.

14. Larsen T, Dalskov S, van Baak M, et al. Diets with high or low protein content and glycemic index for weight-loss maintenance. New Engl J Med 2010;363:2102-2113.

15. Canfi A, Gepner Y, Schwarzfuchs D, et al. Effect of changes in the intake of weight of specific food groups on successful body weight loss during a multi-dietary strategy intervention trial. $\mathrm{J} \mathrm{Am} \mathrm{Coll}$ Nutr 2011;30:491-501.

16. Halton $\mathrm{T}, \mathrm{Hu} \mathrm{F}$. The effects of high protein diets on thermogenesis, satiety and weight loss: A critical review. J Am Coll Nutr 2004;23: 373-385.
17. Rolls B, Hetherington M, Burley V. The specificity of satiety: The influence of foods of different macronutrient content on the development of satiety. Physiol Behav 1988;43:145-153.

18. Holt S, Miller J, Petocz P, et al. A satiety index of common foods. Eur J Clin Nutr 1995;49:675-690.

19. Vander Wal J, Marth J, Khosla P, et al. Short-term effect of eggs on satiety in overweight and obese subjects. J Am Coll Nutr 2005; 24:510-515.

20. Layman D, Walker D. Potential importance of leucine in treatment of obesity and the metabolic syndrome. J Nutr 2006;136(1 Suppl): 319S-323S.

21. Blouet C, Jo Y, Li X, et al. Mediobasal hypothalamic leucine sensing regulates food intake through activation of a hypothalamus-brainstem circuit. J Neurosci 2009;29:8302-8311.

22. Vander Wal J, Gupta A, Khosla P, et al. Egg breakfast enhances weight loss. Int J Obes (Lond) 2008;32:1545-1551.

23. Fallaize R, Wilson L, Gray J, et al. Variation in the effects of three different breakfast meals on subjective satiety and subsequent intake of energy at lunch and evening meal. Eur J Nutr 2013;52: $1353-1359$

24. LaCombe A, Ganji V. Influence of two breakfast meals differing in glycemic load on satiety, hunger, and energy intake in preschool children. Nutr J 2010;12:53.

25. Kuczmarski R, Ogden C, Guo S, et al. 2000 CDC Growth Charts for the United States: Methods and development. Vital Health Stat 2002;246:1-190.

26. Laurent J. Psychometric properties for the Children's Power of Food Scale in a diverse sample of pre-adolescent youth. Appl Nurs Res 2014;28:127-131.

27. Tanofsky-Kraff M, Ranzenhofer L, Yanovski S, et al. Psychometric properties of a new questionnaire to assess eating in the absence of hunger in children and adolescents. Appetite 2008;51:148-155.

28. Cepeda-Benita A, Gleaves D, Williams T, et al. The development and validation of the State and Trait food Cravings Questionnaires. Behav Therapy 2000;31:151-173.

Address correspondence to: Kerri N. Boutelle, PhD Department of Pediatrics University of California, San Diego 8500 La Jolla Village Drive La Jolla, CA 92109

E-mail: kboutelle@ucsd.edu 